\title{
Sequential and Exhaustive Ionization of Analytes with Different Surface Activity by Probe Electrospray Ionization
}

\author{
Mridul Kanti Mandal, ${ }^{1}$ Lee Chuin Chen, ${ }^{2}$ Kenzo Hiraoka ${ }^{1}$ \\ ${ }^{1}$ Clean Energy Research Center, University of Yamanashi, 4-3-11 Takeda, Kofu, Yamanashi, 400-8511, Japan \\ ${ }^{2}$ Interdisciplinary Graduate School of Medicine and Engineering, University of Yamanashi, Kofu, Yamanashi, Japan
}

\begin{abstract}
The probe electrospray ionization (PESI) is an ESI-based ionization technique that generates electrospray from the tip of a solid metal needle. In this work, mass spectra for the single-shot PESI were measured as a function of time for a mixture of several analytes with different surface activity values. It was found that the analytes were elecrosprayed in the order of their surface activity. For example, detergent and protein were detected separately and respectively at the first and last stages of electrospray, for a mixed sample of $10^{-3} \mathrm{M}$ Triton X100 and $10^{-5} \mathrm{M}$ cytochrome $c$. For human breast cancer tissue, at first proteins such as $\alpha$ and $\beta$ chains of hemoglobin, were observed as the dominant ions, but just before the liquid droplet on the needle was depleted only lipids were observed, meaning that PESI has the advantage of the suppression effect with analytes being detected separately in the order of their surface activity values.
\end{abstract}

Key words: Electrospray, Nano-electrospray, Probe electrospray, Surface activity, Suppression effect

\section{Introduction}

$\mathrm{S}$ ince the pioneering work by Fenn and co-workers, ESIM MS has been one of the main tools for analyzing biological compounds [1, 2]. In ESI, the analysis of hydrophilic analytes is always challenging for mixtures that contain different physicochemical properties such as surfaceactive (i.e.; solvophobic) contaminants, as these compounds actively provide ions towards the droplet surface [3]. Nonionic-based surfactants such as Triton X100 always form adducts with proteins and also suppress the analyte ion signals [4]. Supramolecular system-based extraction/separa-

Electronic supplementary material The online version of this article (doi:10.1007/s13361-011-0162-4) contains supplementary material, which is available to authorized users.

Correspondence to: Kenzo Hiraoka; e-mail: hiraoka@yamanashi.ac.jp tions and HPLC techniques are successful in the removal of detergents [5] but they are time-consuming, tedious, and sometimes can produce carryover/memory effects [6]. Not only the surface-active compounds at low concentrations cause severe signal suppression, but also charged solution additives, e.g., $\mathrm{NaCl}$, can suppress the ion signals of analytes with concentrations higher than $1 \mathrm{mM}$ [7-12].

Zhou and Cook have developed a model [13] that accounts qualitatively for the effects of ion pairing, surface activity, and electrophoretic mobility in electrospray mass spectrometry. Their data suggested that high surface concentrations can be readily achieved with surface-active ions. Therefore, a highly hydrophobic compound should have a much higher surface concentration than a more hydrophilic compound, and predominantly hydrophobic compounds will be detected by ESI-MS [13].

In normal ESI using capillaries, sample solutions are supplied continuously through the capillary. Thus, the 
generated charged droplets ejected from the Taylor cone must contain all the components originally contained in the sample solution. Through the off-spring droplet formation of the primary charged droplets, more surface-active analytes are carried away by the progeny droplets and less surfaceactive ones are left in the primary droplets. The less surfaceactive analytes left in the primary droplets have smaller chances for further off-spring droplet formation because of the decrease in excess charges in the primary droplets, i.e., ion signals of less-surface-active analytes are suppressed by the presence of more-surface-active analytes [7, 11, 14].

Recently, several ESI-based ionization techniques have been developed that can avoid the suppression effect that is inherent to normal ESI, e.g., desorption electrospray ionization (DESI) by Cooks and coworkers [15], fused-droplet electrospray ionization (FD-ESI) and electrospray-assisted laser desorption ionization (ELDI) by Shiea and coworkers [16, 17], and extracted electrospray ionization (EESI) by Chen and coworkers [18].

In addition to these new techniques, we have developed the probe electrospray ionization (PESI) [19-25], which uses a solid needle or wire as a sampling probe and as an ESI emitter instead of a capillary. PESI was applied to various biological samples, such as mouse liver, mouse brain, salmon egg, plants, etc. [21-24]. It was found that many components with different surface activity values could be detected simultaneously without any sample preparation. It was also found that PESI was superior to nano-ESI for samples with high concentration $(\geq 100 \mathrm{mM})$ of salts and urea [25]. In PESI, excess charges are continuously provided to the droplet attached to the needle until the liquid is completely sprayed off. During the electrospray in PESI, the more-surface-active analytes are preferentially electrosprayed and the less-surface-active ones are left in the main droplet attached to the needle. However, the less-surfaceactive analytes left in the main droplet could be eventually electrosprayed by the replenishment of excess charges because they are supplied to the main droplet during electrospray due to electrochemical reactions taking place at the interface between the main droplet and the metal surface. Thus, sequential electrospray from the more-surface-active analytes to the less active ones should take place in PESI. This may explain the minor suppression effect observed with PESI [21-24]. The sequential electrospray of analytes with different surface activity values must be reflected on the mass spectra as a function of spray time. In our previous work, we used a $0.1 \mathrm{~mm}$-diameter acupuncture needle. The amount of sample attached on the tip of this needle was about a few pL or less and it was

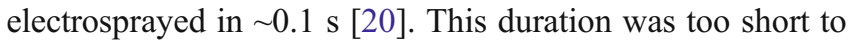
measure the time dependent PESI mass spectra with the time-of-flight (TOF) mass spectrometer used in that work because data processing required $0.1 \mathrm{~s}$. To increase the spraying time, a wire with a larger diameter of $0.5 \mathrm{~mm}$ was used in this work, and this enabled holding a liquid droplet with tens of nanoliters, and the spray time could be increased to tens of seconds, which was sufficient for measuring time- dependent single-shot PESI mass spectra. It was demonstrated that PESI enabled separation of analytes and may be used for analysis of samples containing peptides, lipids, surfactants and/or salts without prior cleanup.

\section{Experimental}

\section{Probe Preparation}

Commercially available titanium (Ti) wire (Nilaco Corporation, Tokyo, Japan) with $0.5 \mathrm{~mm}$ diameter was cut to make the sharp edge at the tip (see Figure 1). When the as-received needle was used as a PESI probe for polar solvents, the liquid attached to the needle formed several tiny droplets on the needle surface, leaving the needle tip dry. The attached tiny liquid droplets flew away from the needle just right after the application of high voltage, and no stable electrospray could be generated. This inconvenience was almost entirely eliminated by treating the $\mathrm{Ti}$ wire surface as described below.

After the Ti wire surface was oxidized under a burner flame, the wire was incubated in $100 \%$ pentafluorophenyltriethoxysilane for $1 \mathrm{~d}$ at room temperature. After incubation, the wire was rinsed with methanol and pure water. By this surface treatment, the needle was reproducibly wet only on the cut side of the needle, as shown in Figure 1. The volume of the attached liquid droplet was estimated to be $20 \sim 50 \mathrm{~nL}$ depending on the samples (see Figure 1).

\section{Probe Electrospray Ionization}

A schematic diagram of the experimental system is shown in Supplementary Figure S1. The experimental procedures were similar to those described in our previous paper [24], except that the droplet was left to be electrosprayed until the electrospray current decreased to zero rather than being periodically renewed. A linear actuator (Citizen, Chiba, Japan) was used to move the Ti wire along a vertical axis perpendicular to the axis of the ion sampling orifice. The stroke distance of the wire was $10 \mathrm{~mm}$. The invasion depth of the needle into the sample was regulated as follows [20, 24]. A shunt resistor $(2 \mathrm{k} \Omega)$ was connected in series with the solid needle at the high voltage side for the electrospray current measurement, and the current signal was amplified and coupled to the low voltage side using cascaded isolation amplifiers (ISO 124P; Texas Instruments, Dallas, Texas, USA). We performed post-amplification and signal conditioning using an RMS-DC converter (AD736; Analog Devices, Norwood, MA, USA), after which the $50 \mathrm{~Hz}$ background signal was used to determine the needle invasion depth. When the needle was not in contact with the sample, a $50 \mathrm{~Hz}$ background current of $\sim 10 \mathrm{nA}$ originating from the power line supply was observed. When the needle tip was just in contact with the sample surface, the background current increased by a factor of $2-3$. This needle position was used as reference to determine the depth of invasion of the needle 

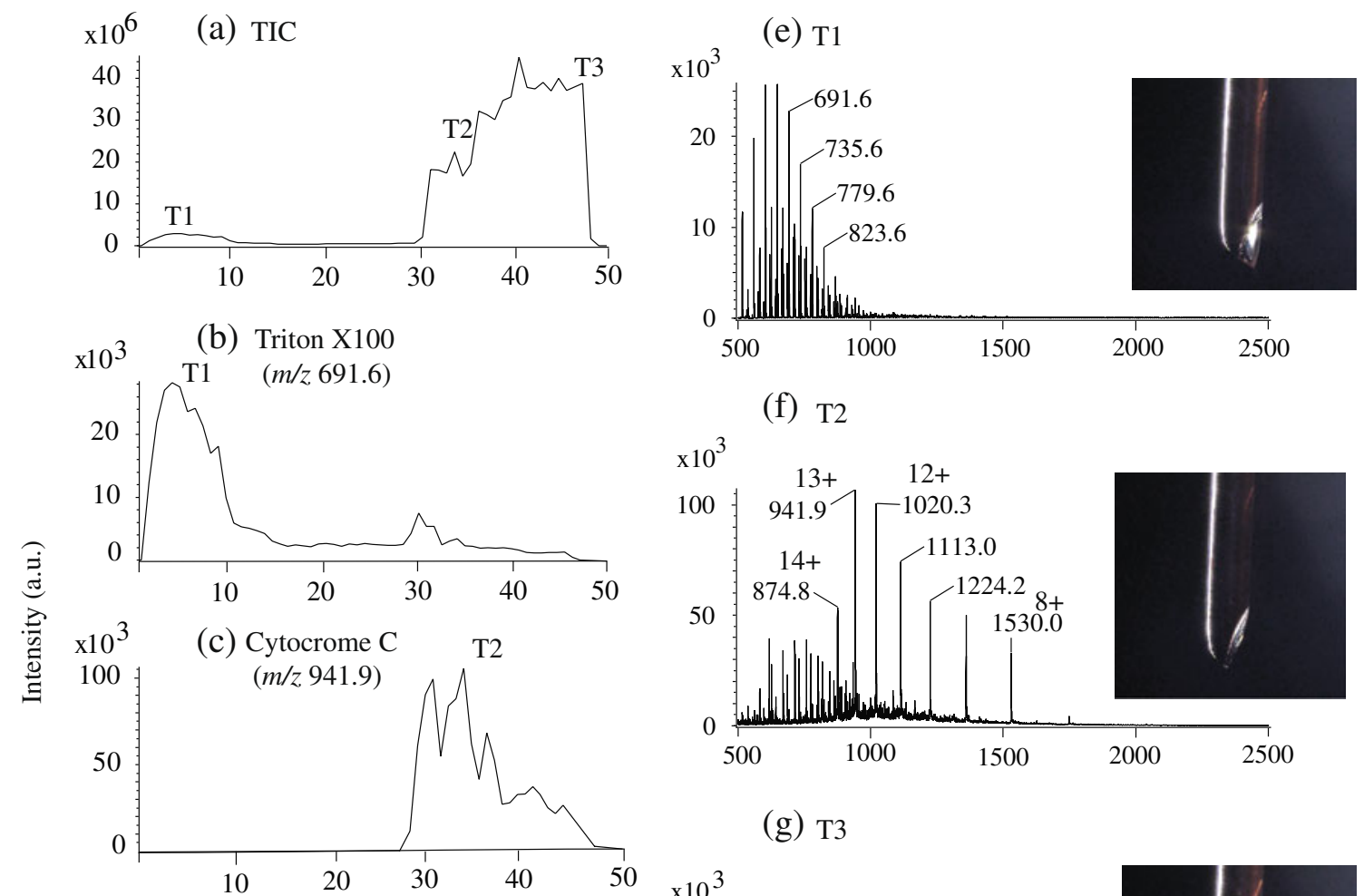

(f) $\mathrm{T} 2$

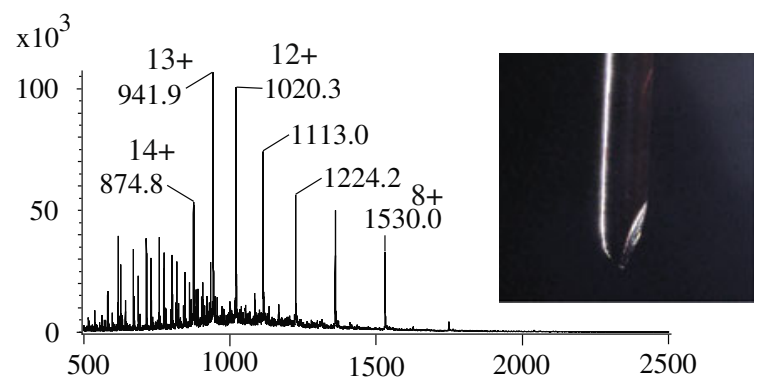

(g) $\mathrm{T} 3$
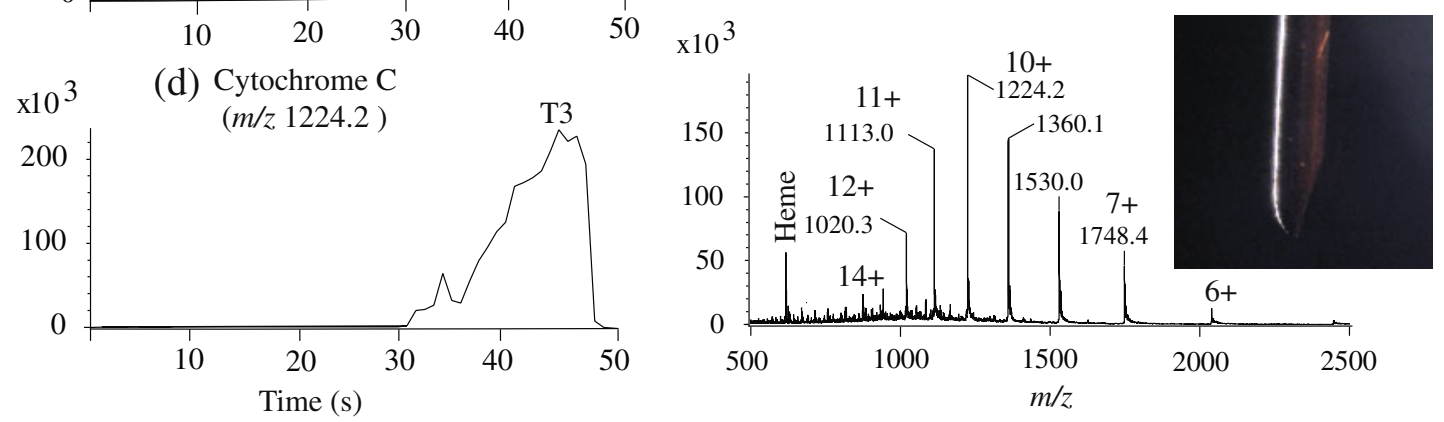

Figure 1. Experimental results for $10^{-3} \mathrm{M}$ Triton $\mathrm{X} 100$ and $10^{-5} \mathrm{M}$ cytochrome $c$ in $\mathrm{H}_{2} \mathrm{O} / \mathrm{MeOH} / \mathrm{HOAc}(74 / 25 / 1)$. (a) Total ion chromatogram (TIC); (b) extracted ion chronogram of Triton X100 at $\mathrm{m} / z 691.6$ [Triton X100 with $n=11+\mathrm{H}]^{+}$; (c) extracted ion chronogram of cytochrome $c$ at $\mathrm{m} / z 941.9$ [cytochrome $c+13 \mathrm{H}]^{13+}$; (d) extracted ion chronogram of cytochrome $c$ at $m / z 1224.2$ [cytochrome $c+10 \mathrm{H}]^{10+}$; (e) Mass spectrum measured at T1. (f) Mass spectrum measured at T2. (g) Mass spectrum measured at T3. Insets in (e), (f), and (g) display the optical microscopic images of the tip of the Ti needle measured at T1, T2, and T3, respectively

into the sample. The invasion depth was adjusted to $0.8 \mathrm{~mm}$ throughout the experiment.

When the wire with the sample droplet on its tip was moved to the highest position, a high voltage of about $3.2-3.8 \mathrm{kV}$ was applied to generate electrospray. The needle tip at the highest position at $2 \mathrm{~mm}$ height and $3 \mathrm{~mm}$ away from the ion sampling orifice gave the strongest ion signals, although the exact position was not very critical. The electrospray was generated in 20 to $50 \mathrm{~s}$ for a single PESI shot depending on the sample. As shown in the inset in Figure 1g, relatively high ion signals were observed even when the liquid on the needle tip became difficult to recognize by the CCD camera. When the electrospray current dropped to zero, the liquid sample might be depleted. The spray time for each sample was reproducible with fluctuation within a few seconds.
The ions generated by PESI were sampled through the ion sampling orifice and transported to the ion guide for collisional cooling and mass-analyzed by an orthogonal-type TOF mass spectrometer (AccuTOF; JEOL, Akishima, Tokyo, Japan). Mass spectra were measured as a function of time during the single-shot PESI. The ions detected by a microchannel plate were converted to digital signals by a 4 $\mathrm{GHz}$ time-to-digital converter. The signal integration time of the digital signal averager was adjusted to $0.5 \mathrm{~s}$.

\section{Sample Preparation}

Bovine cytochrome $c$, gramicidin $\mathrm{S}$, bovine pancreas insulin, L- $\alpha$-phosphatidylcholin (egg yolk), sodium chloride $(\mathrm{NaCl})$, Triton X100, acetic acid (HOAc), and methanol (MeOH) 
were purchased from (Sigma, Tokyo, Japan). Solvents and buffer solutions were HPLC grade, and were used without further purification. Pure water was prepared using a Milli-Q system (Millipore, Bedford, MA, USA). Stock solutions of samples were initially prepared in pure water and then they were diluted with the mixed solvent of $\mathrm{H}_{2} \mathrm{O} / \mathrm{MeOH} / \mathrm{HOAc}$ $(74 / 25 / 1)$. The human breast cancer tissue stored at $-80{ }^{\circ} \mathrm{C}$ was thawed, and a piece $5 \times 5 \times 3 \mathrm{~mm}^{3}$ was cut and rinsed in saline water (aqueous solution of $150 \mathrm{mM} \mathrm{NaCl}$ ) and used for the measurement.

\section{Results and Discussion}

Surfactants are frequently employed to enhance solubilization or to modify the tertiary or quaternary structure of proteins [4]. For example, the nonionic detergent, Triton $\mathrm{X} 100$, is widely used for the solubilization and characterization of the proteins. As mentioned before, in normal ESI the presence of surfactants such as Triton X100 in a protein sample solution leads to severe suppression of less solvophobic proteins [4]. If the sequential electrospray of analytes from more-surface-active analytes to less active ones occurs, the suppression effect could be largely moderated in PESI. In fact, in PESI, sequential electrospray of analytes in the order of surface activity values was observed.

Figure 1a shows the total ion chromatogram (TIC) for the single-shot PESI experiment for $10^{-3} \mathrm{M}$ Triton X100 and $10^{-5} \mathrm{M}$ cytochrome $c$ in $\mathrm{H}_{2} \mathrm{O} / \mathrm{MeOH} / \mathrm{HOAc}(74 / 25 / 1)$. The high voltage $(3.5 \mathrm{kV})$ was applied to the needle at $0 \mathrm{~s}$. TIC shows an initial small increase followed by low ion current, up to $30 \mathrm{~s}$. Then, TIC shows two steep stepfunction-like increases. It is evident that electrospray conditions are changing during the single-shot electrospray (Figure 1a). Figure $1 \mathrm{~b}-\mathrm{d}$ show the extracted ion chronograms of Triton $\mathrm{X} 100$ at $\mathrm{m} / z$ 691.6 [Triton X100 with $n=11+\mathrm{H}]^{+}$, and cytochrome $c$ at $\mathrm{m} / \mathrm{z} 941.9$ [cytochrome $c+13 \mathrm{H}]^{13+}$, and at $\mathrm{m} / \mathrm{z}$ 1224.2 [cytochrome $c+10 \mathrm{H}]^{10+}$, respectively. Figure $1 \mathrm{e}-\mathrm{g}$ display the mass spectra measured at the times $\mathrm{T} 1, \mathrm{~T} 2$, and $\mathrm{T} 3$ denoted in Figure 1a, respectively. The insets in Figure 1e-g show the optical microscopic images of the liquid sample attached on the Ti needle at T1, T2, and T3, respectively.

At T1, only protonated and sodiated Triton X100 were detected in the mass spectrum, but no cytochrome $c$ (Figure 1e). This trend continued just before the steep increase in TIC shown in Figure 1a. The only appearance of Triton X100 in the time interval of $0-30 \mathrm{~s}$ suggests that Triton X100 is being electrosprayed mainly during this period. This must be due to the fact that surface-active Triton X100 is enriched on the liquid droplet surface and cytochrome $c$ was almost totally suppressed by the presence of Triton X100 up to 30 s. Supplementary Figure S2 shows the nano-ESI mass spectrum obtained using $1 \mu \mathrm{m}$ inner diameter capillary (EconoTip; New Objective, Woburn, MA, USA) for the same sample solution. The nano-ESI mass spectrum is almost similar to the PESI mass spectrum measured at T1
(Figure 1e). It is evident that cytochrome $c$ was totally suppressed by Triton X100 in nano-ESI.

At $30 \mathrm{~s}$, sudden appearance of cytochrome $c$ was observed (Figure 1c). At this moment, the signal intensities of Triton X100 also show a small increase (Figure 1b). Apparently the electrospray condition changed at $\sim 30 \mathrm{~s}$. This may be attributed to the decrease in the concentration of the surface-active Triton X resulting in the availability of the liquid surface for the enrichment of the less-surface-active cytochrome $c$. That is, the surface excess charges are being replaced from the most-surface-active Triton X100 to the less-surface-active cytochrome $c$. This dynamic change in the components on the droplet surface is reflected in the total ion chromatogram in Figure 1a. The start of enrichment of cytochrome $c$ on the liquid surface enhanced the efficiency of the electrospray.

From Figure 1, it is clear that for the mixture of two samples Triton X100 was sprayed first and then cytochrome c. Although the concentration of Triton X100 was 100 times that of cytochrome $c$, the ion current of the former, appearing in the range $0-30 \mathrm{~s}$ was much lower than that for cytochrome $c$ appearing at 30-48 s (Figure 1a). Since cytochrome $c$ only appears until virtually almost all Triton X100 is gone, most of the Triton X100 must have left the sample solution as neutral molecules, probably sent off at the early stage of electrospray.

A steep increase followed by gradual decrease was observed for [cytochrome $c+13 \mathrm{H}]^{13+}$ at $\mathrm{m} / z \quad 941.9$ (Figure 1c). In contrast, [cytochrome $c+10 \mathrm{H}]^{10+}$ at $\mathrm{m} / \mathrm{z}$ 1224.2 showed a gradual increase followed by sudden decrease (Figure 1d). Comparison of Figure 1d and g shows that the charge distribution shifted to higher charge states (Figure 1f). Since the charge distribution reflects the higherorder structures of protein, a conformational change of protein seems to have taken place during electrospray. The higher charge distribution at T2 suggests that protein has denatured and more basic sites were exposed to solution. The denaturation at T2 may partly be attributed to the coexistence of Triton X100, which is known to promote the denaturation of proteins. The lower charge distribution in Figure 1g may be explained by the depletion of Triton X100 at the end of electrospray. In normal ESI, protein that was less denatured would have a lower ionization efficiency as it had to be ionized by the charged residue mechanism when there is little excess charge left in the primary droplet, whereas in PESI it could be efficiently ionized because more excess charges are always available.

Juraschek et al. measured nano-ESI mass spectra of insulin from $\mathrm{NaCl}$ solutions by using the self-pulled gold-coated glass capillaries (borosilicate glass, $1.2 \mathrm{~mm}$ o.d.) with orifices of $\leq 2$ $\mu \mathrm{m}$ in diameter [26]. In the mass spectrum for a $10^{-2} \mathrm{M} \mathrm{NaCl}$ and $10^{-5} \mathrm{M}$ insulin in $\mathrm{H}_{2} \mathrm{O} / \mathrm{MeOH} / \mathrm{HOAc}(48 / 48 / 4)$, they observed series of $\left[\mathrm{Na}_{\mathrm{n}} \mathrm{Cl}_{\mathrm{n}-1}\right]^{+}$and $\left[\mathrm{M}+\mathrm{H}_{\mathrm{n}-\mathrm{m}}+\mathrm{Na}_{\mathrm{m}}\right]^{\mathrm{n}+}$ where $\mathrm{M}$ denotes insulin. We also performed PESI measurement for insulin in $\mathrm{NaCl}$ solution. Figure $2 \mathrm{a}-\mathrm{d}$ show the TIC, extracted ion chronograms of insulin $[\mathrm{M}+4 \mathrm{H}]^{4+}$ at $\mathrm{m} / \mathrm{z}$ 1434.5, $[\mathrm{M}+$ 
(a) TIC

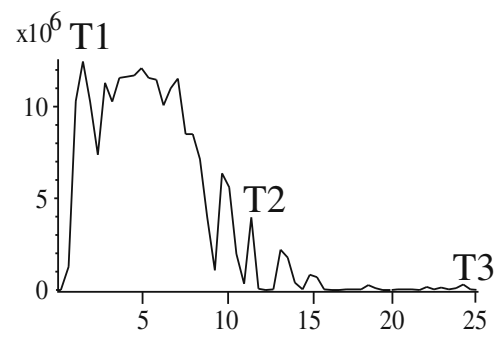

(b) Insulin $(\mathrm{m} / \mathrm{z}$ 1434.5)

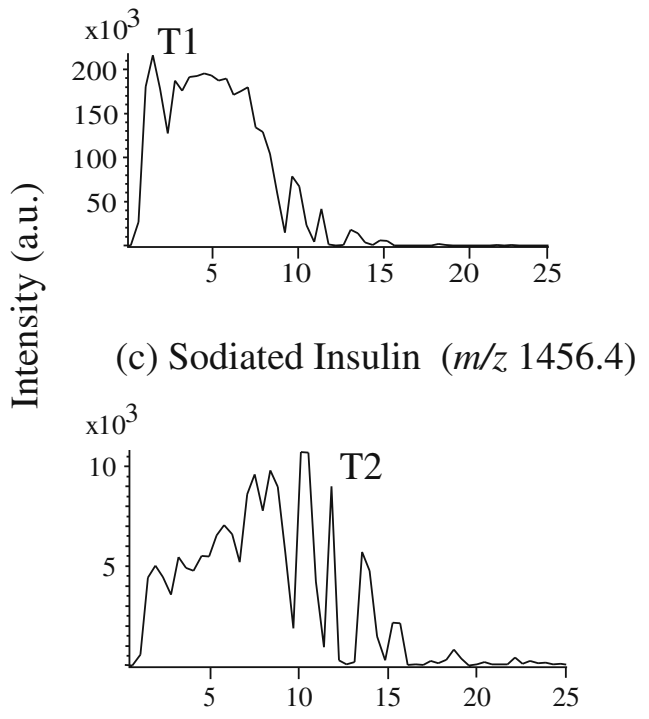

(d) $\mathrm{NaCl}(m / z, 782.5)$

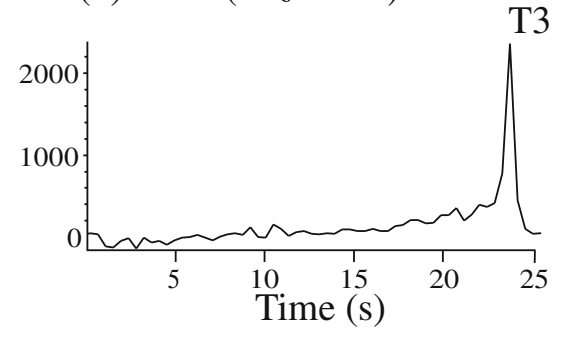

(e) $\mathrm{T} 1$

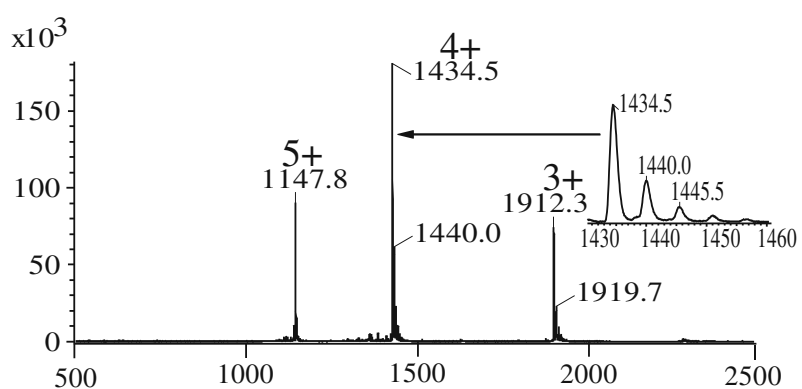

(f) $\mathrm{T} 2$

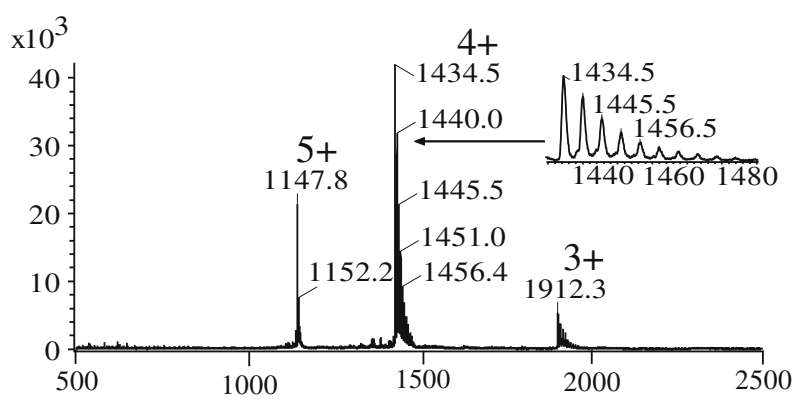

(g) T3

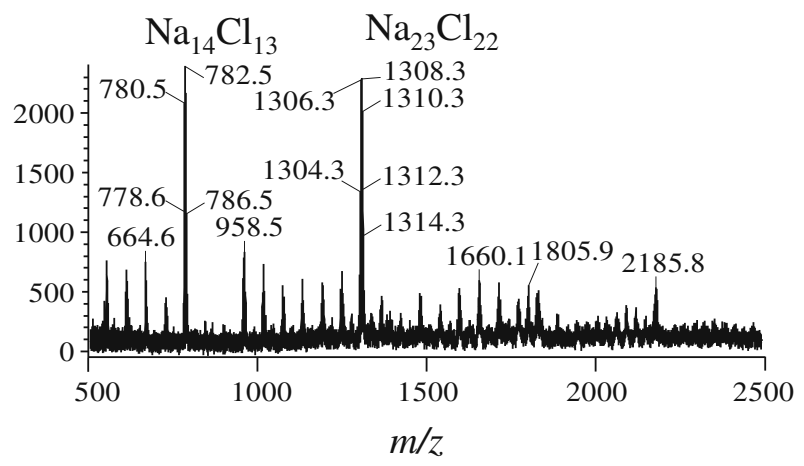

Figure 2. Experimental results for $15 \times 10^{-3} \mathrm{M} \mathrm{NaCl}$ and $10^{-5} \mathrm{M}$ insulin in $\mathrm{H}_{2} \mathrm{O} / \mathrm{MeOH} / \mathrm{HOAc}(74 / 25 / 1)$. (a) Total ion chromatogram (TIC); (b) extracted ion chronogram of insulin $[\mathrm{M}+4 \mathrm{H}]^{4+}$ at $\mathrm{m} / \mathrm{z}$ 1434.5; (c) extracted ion chronogram of insulin $[\mathrm{M}+4 \mathrm{Na}]^{4+}$ at $\mathrm{m} / \mathrm{z}$ 1456.4; (d) extracted ion chronogram of $\left[\mathrm{Na}_{14} \mathrm{Cl}_{13}\right]^{+}$at $m / z$ 782.5. (e) Mass spectrum measured at $\mathrm{T} 1$; (f) mass spectrum measured at T2; (g) mass spectrum measured at T3

$4 \mathrm{Na}]^{4+}$ at $\mathrm{m} / z$ 1456.4, and $\left[\mathrm{Na}_{14} \mathrm{Cl}_{13}\right]^{+}$at $\mathrm{m} / \mathrm{z} 782.5$, respectively, for $1.5 \times 10^{-2} \mathrm{M} \mathrm{NaCl}$ and $10^{-5} \mathrm{M}$ insulin in $\mathrm{H}_{2} \mathrm{O}$ / $\mathrm{MeOH} / \mathrm{HOAc}(74 / 25 / 1)$. Figure $2 \mathrm{e}-\mathrm{g}$ show the PESI mass spectra measured at T1, T2, and T3 shown in Figure 2a. The $\mathrm{NaCl}$ cluster ions $\left[\mathrm{Na}_{\mathrm{n}} \mathrm{Cl}_{\mathrm{n}-1}\right]^{+}$are absent in Figure 2e and $\mathrm{f}$, suggesting that $\mathrm{NaCl}$ is being enriched in the main droplet with time. In fact, the value of $\mathrm{m}$, the number in $\left[\mathrm{M}+\mathrm{H}_{4-\mathrm{m}}+\mathrm{Na}_{\mathrm{m}}\right]^{4+}$, increases with time $\mathrm{T} 1 \rightarrow \mathrm{T} 2$ because of the increase in $\mathrm{NaCl}$ concentration in the droplet. The ion chronogram for $\left[\mathrm{Na}_{14} \mathrm{Cl}_{13}\right]^{+}$at $m / z 782.5$ (Figure 2d) shows a sharp increase at $\mathrm{T} 3$, indicating that $\mathrm{NaCl}$ is electrosprayed mainly at the last stage of the electrospray. At $\mathrm{T} 3$, only $\mathrm{NaCl}$ cluster ions and no ions due to insulin are observed (Figure $2 \mathrm{~g}$ ). In this binary sample, almost complete separation of peptide and salt takes place. In other words, all of the insulin may have been electrosprayed before $\mathrm{NaCl}$ clusters appear. This result confirms the ionization of solution components in the order of decreasing surface activity but also the efficient ionization of the less-surface-active components, which would not occur with normal electrospray.

To examine the applicability of PESI to biological samples containing proteins and lipids, a mixture of protein and phosphatidylcholine was examined. Figure 3 summarizes the PESI experimental results dealing with the mixture 
(a) TIC
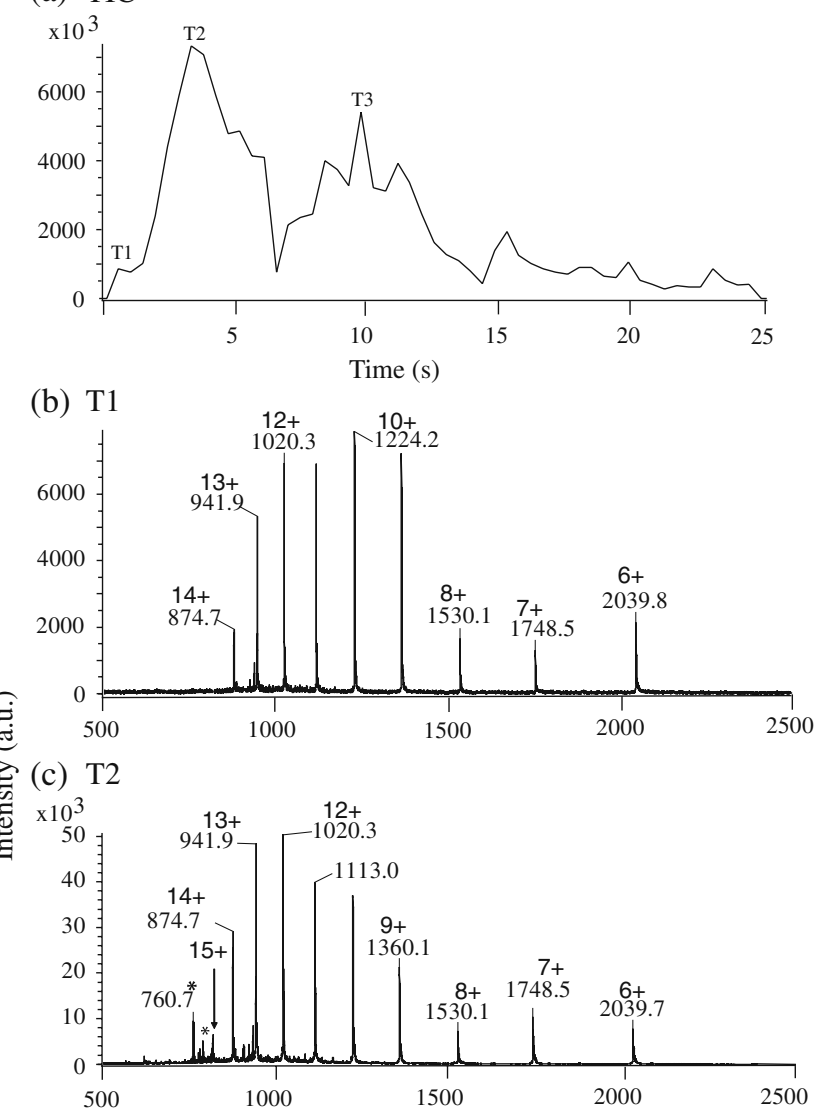

(d) $\mathrm{T} 3$

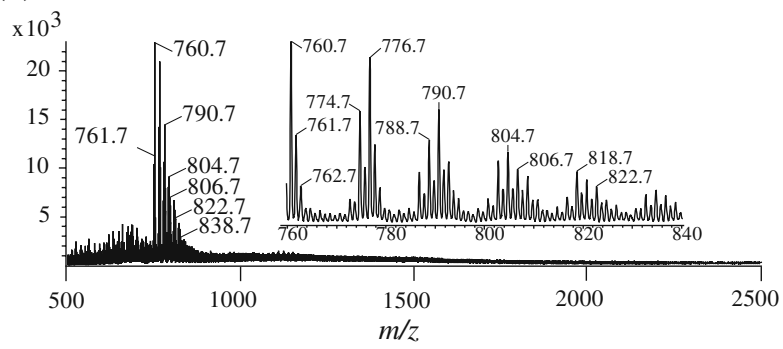

Figure 3. Experimental results for $10^{-5} \mathrm{M}$ cytochrome $c$ and $10^{-5} \mathrm{M} \mathrm{L- \alpha -phosphatidylcholines} \mathrm{(egg} \mathrm{yolk)} \mathrm{in} \mathrm{H}_{2} \mathrm{O} / \mathrm{MeOH}$ / HOAc (74/25/1). (a) Total ion chromatograms (TIC); (b) mass spectrum measured at $\mathrm{T} 1$; (c) mass spectrum measured at T2; (d) mass spectrum measured at T3. Peaks assigned with asterisk $\left(^{*}\right)$ shown in (c) originate from L- $\alpha$-phosphatidylcholines

of $10^{-5} \mathrm{M}$ cytochrome $c$ and $10^{-5} \mathrm{M}$ L- $\alpha$-phosphatidylcholines in $\mathrm{H}_{2} \mathrm{O} / \mathrm{MeOH} / \mathrm{HOAc}(74 / 25 / 1)$. Figure $3 \mathrm{a}-\mathrm{d}$ display the TIC, mass spectra at T1, T2, and T3 denoted in Figure 3a, respectively. At $\mathrm{T} 1$, the onset of the electrospray, only cytochrome $c$ was observed. At T2, in the middle of electrospray, the mass spectrum was slightly contaminated by L- $\alpha$ phosphatidylcholines but cytochrome $c$ was still dominant. However, at T3 and at later stages of electrospray, only L- $\alpha$ phosphatidylcholines but no protein was observed, clearly indicating that cytochrome $c$ and L- $\alpha$-phosphatidylcholines were separately detected by PESI, although both components were observed in the middle of the electrospray (Figure 3a).

Finally, the results of the application of PESI to a realworld sample, a tissue of human breast cancer are presented. (A slice of a tumor of size $5 \times 5 \times 3 \mathrm{~mm}^{3}$ was prepared as described in the Experimental section). The PESI mass spectra were measured immediately after $10 \mu \mathrm{L}$ of mixed solvent of $\mathrm{H}_{2} \mathrm{O} / \mathrm{MeOH} / \mathrm{HOAc}(50 / 50 / 1)$ was dropped on the surface of tumor tissue. The inset in Figure $4 \mathrm{a}$ shows the TIC that has three peaks. Figure $4 \mathrm{a}-\mathrm{c}$ display the mass spectra at T1, T2, and T3 denoted in the inset in Figure 4a. Ion signals due to heme, $\alpha$ and $\beta$ chains of hemoglobin, and lipids in the range of $m / z$ 700-900 (denoted with asterisks and circles) were observed (Figure 4). In a separate experiment, some of the lipids appearing in Figure 4 were assigned
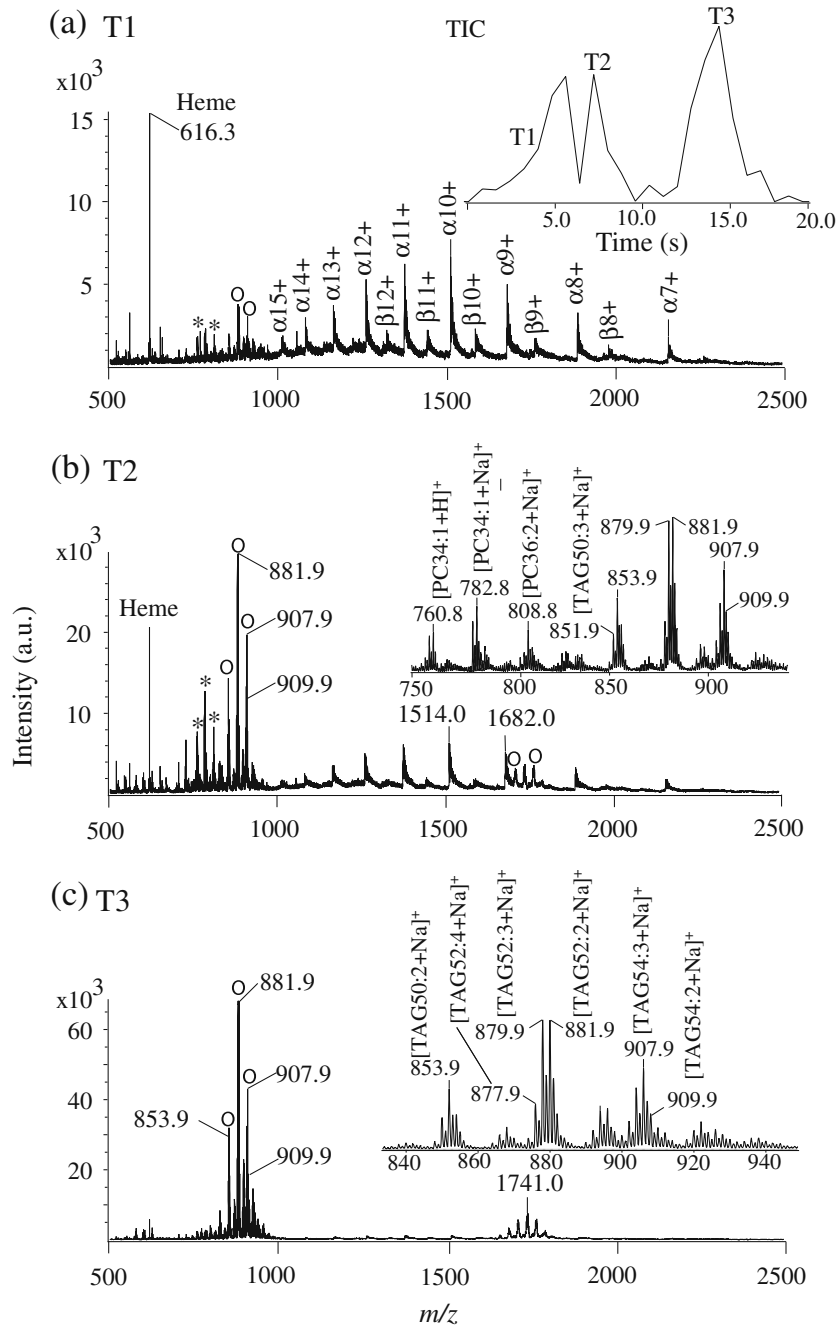

Figure 4. Mass spectra for a human breast cancer tissue (a) Mass spectrum measured at T1; inset: total ion chromatogram; (b) mass spectrum measured at T2; inset: expanded mass spectrum at $\mathrm{m} / \mathrm{z}$ 750-950; (c) mass spectrum measured at T3; inset: expanded mass spectrum at $\mathrm{m} / \mathrm{z}$ 840-940. Asterisks $\left(^{*}\right)$ and open circles (०) stand for peaks from phosphatidylcholin (PC) and sodiated triacylglycerides (TAG) 
by exact mass analysis using a high-resolution mass spectrometer (Orbitrap Exactive, Thermo, Bremen, Germany). The peaks denoted with asterisks and those denoted with circles were assigned to be phosphatidylcholines (PC) and triacylglycerides (TAG), as shown in insets in Figure $4 b$ and $c)$. At the initial stage of electrospray (T1), heme and $\alpha$ and $\beta$ chains of hemoglobin were observed as the major ions with weaker PC and TAG signals. With time $\mathrm{T} 1 \rightarrow \mathrm{T} 2$, the intensity of ion signals due to hemoglobin weaken and these signals are taken over by those of PC and TAG. Ion signals of PC are observed at T1 and T2, but they decrease to the noise level at T3. Instead, sodiated TAGs become predominant at the latest stage of electrospray. That is, separation of components takes place in the order of hemoglobin $\rightarrow \mathrm{PC} \rightarrow \mathrm{TAG}$ under the present experimental conditions. To us, it is surprising that highly hydrophobic TAGs are detected at this late stage of electrospray. Even though the denatured proteins should become more hydrophobic than their intact forms, we could not understand why the sample components were separated in the order of protein $\rightarrow$ TAG. More extensive work is underway in our laboratory to elucidate the phenomena taking place in the single-shot PESI.

\section{Conclusion}

In the present work, the single-shot PESI was examined using a titanium wire with $0.5 \mathrm{~mm}$-diameter. By modifying the wire surface with pentafluorophenyl-triethoxysilane to be hydrophobic, it was possible to electrospray liquid samples of $20-50 \mathrm{~nL}$ attached on the needle tip for periods of tens of seconds. In normal electrospray, the main part of the droplet is charge-depleted by the spawning the highly-charged microdroplets. In contrast, the main part of the droplet remains attached to the electrode in PESI and, thus, it is not charge limited. Because the droplet is allowed to be electrosprayed to depletion, single-shot PESI may be the way to analyze virtually all ionizable components in the sample. It was found that analytes with different surface activities were detected sequentially in the order of their surface activity. Because single-shot PESI does not need any special sample pretreatment, it may be applicable to the quick survey of wide-range real-world biological samples. One drawback of PESI is that the analyte signal intensity changes significantly as a factor not just of time, but also of solution composition. The relative quantitative comparisons for analytes remain to be investigated.

\section{Acknowledgement}

The authors are grateful to Professors S. Takeda, H. Fujii, and $\mathrm{K}$. Yoshimura of the University of Yamanashi for giving them the sample of human breast cancer tissue. This work was supported by the Development of System and Technology for Advanced Measurement and Analysis Program from the Japan
Science and Technology Agency. The authors are greatly indebted to the reviewers for valuable comments in the improvement of the manuscript.

\section{References}

1. Fenn, J., Mann, M., Meng, C., Wong, S., Whitehouse, C.: Electrospray ionization for mass spectrometry of large biomolecules. Science 246, 64-71 (1989)

2. Fenn, J.B.: Mass spectrometric implications of high-pressure ion sources. Int. J. Mass Spectrom 200, 459-478 (2000)

3. Schmidt, A., Karas, M., Dülcks, T.: Effect of different solution flow rates on analyte ion signals in nano-ESI MS, or: when does ESI turn into nano-ESI? J. Am. Soc. Mass Spectrom 14, 492-500 (2003)

4. Loo, R.R., Dales, N., Andrews, P.C.: Surfactant effects on protein structure examined by electrospray ionization mass spectrometry. Protein Sci 3, 1975-1983 (1994)

5. Merino, F., Rubio, S., Pérez-Bendito, D.: Supramolecular systemsbased extraction-separation techniques coupled to mass spectrometry. $J$. Sep. Sci 28, 1613-1627 (2005)

6. Mitulovic, G., Stingl, C., Steinmacher, I., Hudecz, O., Hutchins, J.R.A., Peters, J., Mechtler, K.: Preventing Carryover of Peptides and Proteins in Nano LC-MS Separations. Anal. Chem 81, 5955-5960 (2009)

7. Enke, C.G.: A Predictive Model for Matrix and Analyte Effects in Electrospray Ionization of Singly-Charged Ionic Analytes. Anal. Chem 69, 4885-4893 (1997)

8. Constantopoulos, T.L., Jackson, G.S., Enke, C.G.: Effects of salt concentration on analyte response using electrospray ionization mass spectrometry. J. Am. Soc. Mass Spectrom 10, 625-634 (1999)

9. Cech, N.B., Enke, C.G.: Relating electrospray ionization response to nonpolar character of small peptides. Anal. Chem 72, 2717-2723 (2000)

10. Cech, N.B., Krone, J.R., Enke, C.G.: Predicting electrospray response from chromatographic retention time. Anal. Chem 73, 208-213 (2001)

11. Cech, N.B., Enke, C.G.: The effect of affinity for charged droplet surfaces on the fraction of analyte charged in the electrospray process. Anal. Chem 73, 4632-4639 (2001)

12. Haddrell, A.E., Agnes, G.R.: Organic Cation Distributions in the Residues of Levitated Droplets with Net Charge: Validity of the Partition Theory for Droplets Produced by an Electrospray. Anal. Chem 76, 53-61 (2004)

13. Zhou, S., Cook, K.D.: A mechanistic study of electrospray mass spectrometry: charge gradients within electrospray droplets and their influence on ion response. J. Am. Soc. Mass Spectrom 12, 206-214 (2001)

14. Kebarle, P., Verkerk, U.H.: Electrospray: From ions in solution to ions in the gas phase, what we know now. Mass Spectrom. Rev 28, 898-917 (2009)

15. Cooks, R.G., Ouyang, Z., Takats, Z., Wiseman, J.M.: Ambient Mass Spectrometry. Science 311, 1566-1570 (2006)

16. Chang, D., Lee, C., Shiea, J.: Detecting Large Biomolecules from HighSalt Solutions by Fused-Droplet Electrospray Ionization Mass Spectrometry. Anal. Chem 74, 2465-2469 (2002)

17. Shiea, J., Huang, M., Hsu, H., Lee, C., Yuan, C., Beech, I., Sunner, J.: Electrospray-assisted laser desorption/ionization mass spectrometry for direct ambient analysis of solids. Rapid Commun. Mass Spectrom 19, 3701-3704 (2005)

18. Chen, H., Venter, A., Cooks, R.G.: Extractive electrospray ionization for direct analysis of undiluted urine, milk and other complex mixtures without sample preparation. Chem. Commun 19, 2042 (2006)

19. Hiraoka, K., Nishidate, K., Mori, K., Asakawa, D., Suzuki, S.: Development of probe electrospray using a solid needle. Rapid Commun. Mass Spectrom 21, 3139-3144 (2007)

20. Chen, L.C., Nishidate, K., Saito, Y., Mori, K., Asakawa, D., Takeda, S., Kubota, T., Hori, H., Hiraoka, K.: Characteristics of probe electrospray generated from a solid needle. J. Phys. Chem. B 112, 11164-11170 (2008)

21. Chen, L.C., Nishidate, K., Saito, Y., Mori, K., Asakawa, D., Takeda, S., Kubota, T., Terada, N., Hashimoto, Y., Hori, H., Hiraoka, K.: Application of probe electrospray to direct ambient analysis of 
biological samples. Rapid Commun. Mass Spectrom 22, 2366-2374 (2008)

22. Yoshimura, K., Chen, L.C., Asakawa, D., Hiraoka, K., Takeda, S.: Physical properties of the probe electrospray ionization (PESI) needle applied to the biological samples. J. Mass Spectrom 44, 978-985 (2009)

23. Yu, Z., Chen, L.C., Suzuki, H., Ariyada, O., Erra-Balsells, R., Nonami, H., Hiraoka, K.: Direct profiling of phytochemicals in tulip tissues and in vivo monitoring of the change of carbohydrate content in tulip bulbs by probe electrospray ionization mass spectrometry. J. Am. Soc. Mass Spectrom 20, 2304-2311 (2009)
24. Chen, L.C., Yoshimura, K., Yu, Z., Iwata, R., Ito, H., Suzuki, H., Mori, K., Ariyada, O., Takeda, S., Kubota, T., Hiraoka, K.: Ambient imaging mass spectrometry by electrospray ionization using solid needle as sampling probe. J. Mass Spectrom 44, 1469-1477 (2009)

25. Mandal, M.K., Chen, L.C., Hashimoto, Y., Yu, Z., Hiraoka, K.: Ditection of biomolecules from solutions with high concentration of salts using probe electrospray amd nano-electrospray ionization mass spectrometry. Anal. Methods 2, 1905-1912 (2010)

26. Juraschek, R., Dulcks, T., Karas, M.: Nanoelectrospray-more than just a minimized-flow electrospray ionization source. J. Am. Soc. Mass Spectrom 10, 300-308 (1999) 